\title{
Longitudinal White Matter Changes following Carbon Monoxide Poisoning: A 9-Month Follow-Up Voxelwise Diffusional Kurtosis Imaging Study
}

\author{
(1DM.-C. Chou, (1D).-Y. Li, and (1DP.-H. Lai
} O.

\begin{abstract}
BACKGROUND AND PURPOSE: Patients with carbon monoxide (CO) intoxication exhibit progressive WM changes that are not wellunderstood. The purpose of this study was to detect longitudinal WM changes using voxelwise diffusional kurtosis imaging in patients with $\mathrm{CO}$ intoxication from the acute-to-chronic stage after $\mathrm{CO}$ intoxication.
\end{abstract}

MATERIALS AND METHODS: Twenty-four patients with CO intoxication and 21 age- and sex-matched healthy controls were enrolled in this study. Diffusional kurtosis imaging was performed on all subjects and was conducted repeatedly in patients at 1 week and 1, 3, and 9 months after CO intoxication. Voxelwise diffusional kurtosis imaging analysis was performed to detect global WM changes in the patients with and without WM lesions. Receiver operating characteristic analysis was performed to compare the performance of diffusional indices in differentiating patients with delayed neuropsychiatric sequelae from patients without them.

RESULTS: In voxelwise analysis, progressive WM changes were detected in patients with WM lesions. In the acute phase, WM injuries were found mainly in the dopaminergic pathways at 1 week, whereas in the chronic stage, WM injuries extended toward subcortical areas from 1 to 9 months. However, no significant WM change was noted in patients without WM lesions during the 9 months after CO intoxication. Moreover, receiver operating characteristic analysis demonstrated that axial kurtosis and mean kurtosis values had better performance than other diffusional indices in differentiating patients with delayed neuropsychiatric sequelae from patients without them at 1 week after CO intoxication.

CONCLUSIONS: Voxelwise diffusional kurtosis imaging analysis was helpful to longitudinally investigate WM changes and predict the prognosis of patients after $\mathrm{CO}$ intoxication.

ABBREVIATIONS: $A D=$ axial diffusivity; $A K=$ axial kurtosis; $C O=$ carbon monoxide; $C P=$ cerebral peduncle; $D K I=$ diffusional kurtosis imaging; $D N S=$ delayed neuropsychiatric sequelae; $F A=$ fractional anisotropy; $F W M=$ frontal white matter; $G P=$ globus pallidus; $I C=$ internal capsule; $M D=$ mean diffusivity; $M K=$ mean kurtosis; $\mathrm{RD}$ = radial diffusivity; RK = radial kurtosis; $\mathrm{WML}=$ white matter lesion

C arbon monoxide (CO) intoxication is an aggravating health issue that can cause coma or death following accidental inhalation or attempted suicide. Survivors of carbon monoxide intoxication may exhibit acute or chronic neurologic or psychological problems that can dramatically impact their daily activities. ${ }^{1,2}$ Hyperbaric oxygen therapy is usually performed at the acute stage

Received October 10, 2018; accepted after revision January 14, 2019.

From the Departments of Medical Imaging and Radiological Sciences (M.-C.C.), Healthcare Administration and Medical Informatics (M.-C.C.), Kaohsiung Medical University, Kaohsiung, Taiwan; and Medical Research (M.-C.C.), Kaohsiung Medical University Hospital, Kaohsiung, Taiwan; Department of Neurology (J.-Y.L.), E-Da Hospital, Kaohsiung, Taiwan; School of Medicine (J.-Y.L.), I-Shou University, Kaohsiung, Taiwan; Department of Nursing (J.-Y.L.), Yuh-Ing Junior College of Health Care and Management, Kaohsiung, Taiwan; Department of Radiology (P.-H.L.), Kaohsiung Veterans General Hospital, Kaohsiung, Taiwan; and Faculty of Medicine (P.-H.L.), College of Medicine, National Yang-Ming University, Taipei, Taiwan.

The study has been supported by the Ministry of Science and Technology research grants (106-2314-B-075B-002-MY3 and 101-2314-B-037-047-MY2). to reduce carboxyhemoglobin concentration in the blood and to mitigate neurologic and psychological symptoms. ${ }^{1-4}$ However, delayed neuropsychiatric sequelae (DNS) were observed even after a series of hyperbaric oxygen therapy treatments in patients with CO intoxication. ${ }^{3,5}$

Because patients with $\mathrm{CO}$ intoxication with neuropsychiatric symptoms commonly exhibit WM abnormalities, many studies performed DTI analysis to demonstrate relationships between WM injuries following CO intoxication and delayed encephalopathy, ${ }^{6,7}$ cognitive functions, ${ }^{8-11}$ neuropsychologi-

Please address correspondence to Ping-Hong Lai, MD, Department of Radiology, Kaohsiung Veterans General Hospital, Faculty of Medicine, National Yang-Ming University, 386, Dazhong 1st Rd, Zuoying District, Kaohsiung, 81362, Taiwan; e-mail: pinghonglai@gmail.com

-- Indicates open access to non-subscribers at www.ajnr.org

I. Indicates article with supplemental on-line photos.

http://dx.doi.org/10.3174/ajnr.A5979 

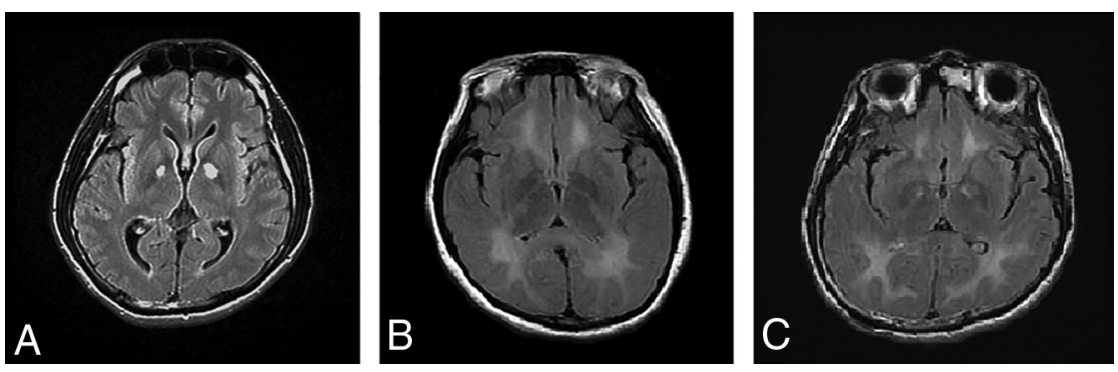

FIG 1. The FLAIR images of 3 patients acquired at 1 month after $C O$ intoxication. $A$, A male patient (32 years of age) who had only GP lesions did not develop DNS. B, A female patient (37 years of age) who had only diffuse WMLs developed DNS. C, A male patient (49 years of age) who had both diffuse WMLs and GP lesions developed DNS.

cal scores, ${ }^{8,9}$ and myelin basic protein levels. ${ }^{12,13}$ However, DTI assumes a Gaussian distribution for water diffusion and cannot accurately estimate diffusion heterogeneity (nonGaussian diffusion) of biologic tissues. ${ }^{14}$ In contrast, diffusional kurtosis imaging (DKI), which quantifies the nonGaussianity of water diffusion, was demonstrated to reflect the microstructural complexity of biologic tissues ${ }^{15,16}$ and to be a potential early biomarker of the neurodegenerative process. ${ }^{17}$ Recent studies further used DKI to detect early WM injuries in patients with $\mathrm{CO}$ intoxication. ${ }^{18,19}$

However, these patients may exhibit delayed encephalopathy and neuropsychiatric symptoms after a lucid period ${ }^{20}$; therefore, it is important to understand the longitudinal WM changes that predict the prognosis of patients with $\mathrm{CO}$ intoxication. A previous study performed longitudinal DTI analysis and demonstrated progressive WM changes $3-10$ months after $\mathrm{CO}$ exposure. ${ }^{9}$ Another study performed longitudinal DKI analysis and showed that DKI better characterized brain damage in 1 patient from 6 days to 20 weeks after $\mathrm{CO}$ intoxication, ${ }^{21}$ but it remains unclear how WM change progresses from acute-tochronic stages and whether more accurate DKI indices can help in the early prediction of prognosis. Therefore, the purposes of this study were to investigate the longitudinal WM changes in patients with $\mathrm{CO}$ intoxication using voxelwise DKI analysis and predict their prognoses from early WM injuries detected 1 week after $\mathrm{CO}$ intoxication using receiver operating characteristic analysis.

\section{MATERIALS AND METHODS}

This prospective study was approved by the local institutional review board of Kaohsiung Veterans General Hospital. This study included 24 patients with $\mathrm{CO}$ intoxication $(\mathrm{M} / \mathrm{F}$ ratio $=11: 13$; mean age $=39.1 \pm 13.2$ years $)$ and 21 age- and sex-matched healthy controls $(\mathrm{M} / \mathrm{F}$ ratio $=10: 11$; mean age $=41.0 \pm 11.9$ years). The exclusion criteria included a history of major neurologic or psychiatric disorders, pregnancy, a metal implant, and claustrophobia. Because delayed sequelae are commonly associated with WM changes, the patients with $\mathrm{CO}$ intoxication were divided into 2 subgroups based on the presence or absence of WM lesions (WMLs) observed within 9 months of follow-up. The WMLs were defined as diffusive, symmetric, or asymmetric hyperintense areas in the deep WM tissues of both cerebral hemispheres on FLAIR images as demonstrated in Fig 1. The patients who exhibited WMLs at any stage of the follow-up were categorized as the WML group and others as the non-WML group. In addition, all patients with $\mathrm{CO}$ intoxication were evaluated by an experienced neurologist regularly at 1 week and 1,3 , and 9 months. This study watched for newly developed neurologic symptoms and signs, including motor deficits, cognitive impairment, dysphagia, dysarthria, gait disturbance, Parkinsonism, seizures, psychosis, and mood disorders. DNS was defined as the appearance of neurologic sequelae after a lucid period of variable duration (usually $2-4$ weeks after CO intoxication). ${ }^{20}$

\section{MR Imaging Acquisition}

MR imaging was performed on a $1.5 \mathrm{~T}$ MR imaging scanner (Signa HDxt, GE Healthcare, Milwaukee, Wisconsin) with an 8-channel head coil. After T1WI, T2WI, and FLAIR imaging were acquired, whole-brain DKI data were acquired using a twice-refocused spin-echo diffusion-weighted pulse sequence with TR/TE $=10,000 / 91 \mathrm{~ms}$, b-values $=1000$ and $2000 \mathrm{~s} / \mathrm{mm}^{2}$, number of $b_{0}$ images $=4$, acceleration factor $=2$, number of diffusion directions $=30$, matrix size $=80 \times 80, \mathrm{FOV}=240 \times$ $240 \mathrm{~mm}$, slice thickness $=3 \mathrm{~mm}$ (isotropic resolution), and number of excitations $=1$. The scan time for DKI was about 10 minutes.

\section{Voxel-Based DKI Analysis}

All imaging data were postprocessed using FSL (http://www. fmrib.ox.ac.uk/fsl), Diffusion Kurtosis Estimator (NeuroImaging Tools and Resources Collaboratory; https://www. nitrc.org/projects/dke/), and SPM12 software (http://www.fil. ion.ucl.ac.uk/spm/software/spm12). First, DKI data were corrected for motion and eddy current distortions using rigid-body and affine registrations, respectively. Second, the FSL Brain Extraction Tool (http://fsl.fmrib.ox.ac.uk/fsl/fslwiki/BET) was used to remove nonbrain signals. Third, the Diffusion Kurtosis Estimator tool was used to calculate axial diffusivity (AD), radial diffusivity (RD), mean diffusivity (MD), fractional anisotropy (FA), axial kurtosis $(\mathrm{AK})$, radial kurtosis (RK), and mean kurtosis $(\mathrm{MK})$. In DTI-related indices, $\mathrm{AD}$ and $\mathrm{RD}$ are the diffusivities parallel and perpendicular to axons, respectively, whereas MD is the averaged diffusivity of a diffusion tensor. FA is the diffusion anisotropy that reflects tissue integrity. In DKI-related indices, AK and RK are the diffusional kurtosis values (non-Gaussianity) parallel and perpendicular to axons, respectively, whereas MK is the averaged kurtosis of a diffusion kurtosis tensor. Finally, whole-brain FA maps were spatially normalized to an International Consortium for Brain Mapping FA template, ${ }^{22}$ which was constructed by normalizing the FA maps of 81 healthy subjects into an International Consortium for Brain Mapping 152-T1 template, using both linear affine and nonlinear demon registrations to minimize global and local differences between individual and template images, respectively. The displacement maps generated from the previous steps were applied to spatially transform the corresponding diffusion maps. 


\begin{tabular}{lccc}
\hline & \multicolumn{2}{c}{$\begin{array}{c}\text { Patients with } \\
\text { CO Intoxication }\end{array}$} & \\
\cline { 2 - 3 } & WML & Non-WML & Controls \\
\hline Sex (M/F) & $6: 5$ & $5: 8$ & $10: 11$ \\
Age (mean) (yr) & $44.5 \pm 15.3$ & $35.7 \pm 9.3$ & $41.0 \pm 11.9$ \\
Duration of coma (mean) (hr) & $44.7 \pm 48.8^{\text {a }}$ & $11.7 \pm 17.6^{\text {a }}$ & NA \\
Carboxyhemoglobin (mean) (\%) & $22.3 \pm 20.9$ & $31.37 \pm 15.1$ & NA \\
Sessions of HBOT (mean) & $11.2 \pm 10.1$ & $8.9 \pm 2.5$ & NA \\
DNS (No.) (M/F) & $5(3: 2)$ & 0 & NA \\
GP involvement (No.) (M/F) & $8(5: 3)$ & $4(2: 2)$ & NA \\
\hline
\end{tabular}

Note:- - HBOT indicates hyperbaric oxygen therapy; NA, not applicable.

${ }^{a} P<.05$
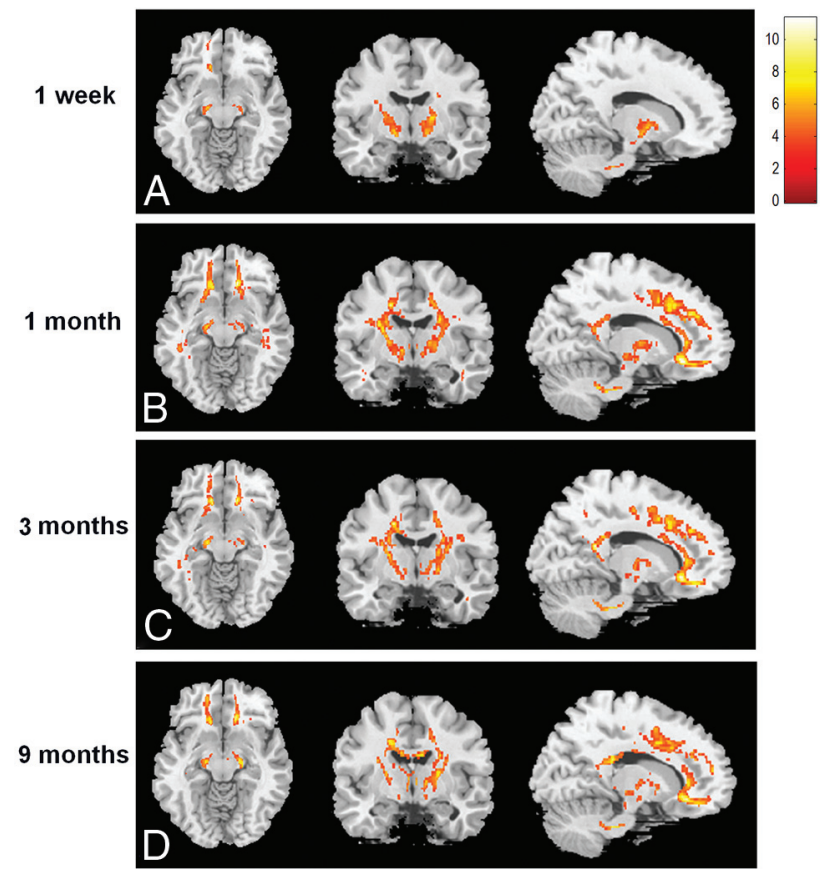

FIG 2. The voxelwise comparison of FA values between patients with WMLs and healthy controls. The yellow-to-red areas indicate regions with values that were significantly decreased in the patients, and the color bar on the right-hand side indicates $T$ values.

\section{Statistical Analysis}

For clinical data, a nonparametric Mann-Whitney $U$ test was performed to examine the difference between WML and non-WML groups, and the results were considered significant with $P<.05$. For DKI data, the voxelwise comparisons between patients and healthy subjects were performed using a 2-sample $t$ test with age and sex as covariates to null the aging and sex effects. The difference was considered significant with cluster-level-corrected $P<$ .05 (uncorrected $P<.001$ and cluster $>100$ voxels). Moreover, receiver operating characteristic analysis was performed for all patients with $\mathrm{CO}$ intoxication to compare the performance of diffusion indices in differentiating patients with and without DNS at 1 week after $\mathrm{CO}$ intoxication.

\section{RESULTS}

\section{Demographic Characteristics}

The demographic characteristics of the patients with CO intoxication and control subjects are listed in Table 1. There were no significant differences in age, sex, hyperbaric oxygen therapy ses- sions, or carboxyhemoglobin levels between the WML and non-WML groups. However, the WML group underwent a significantly longer duration of coma than the non-WML group. Of the 24 patients with CO intoxication, 11 developed visible WMLs within 9 months of follow-up. Most patients with WMLs developed them at 1 month, but 3 patients developed them at only 1 week after CO intoxication. None of the nonWML group had DNS, but 5 of the 11 patients with WMLs had DNS after a lucid period of 3-6 weeks (mean, 30.2 \pm 7.33 days). Moreover, 12 patients had lesions of the globus pallidus (GP); only 3 had DNS, and 8 developed visible WMLs. However, 5 patients with WMLs and 1 without them did not undergo MR imaging at 1 week due to severe coma; 1 patient with WMLs and 1 without them did not undergo MR imaging at 3 months due to loss of contact; and 4 patients with WMLs and 3 without them did not undergo MR imaging at 9 months due to loss of contact or drop-out.

\section{Longitudinal Changes of DTI and DKI Indices}

In patients with WMLs, the DTI indices were significantly altered in the pons, bilateral cerebral peduncle (CP) anterior to the substantia nigra, bilateral internal capsule (IC) next to the thalamus and striatum, bilateral forceps minor, left superior corona radiata, and genu of corpus callosum at 1 week after $\mathrm{CO}$ intoxication, as shown in Figs 2 and On-line Figs 1-3. At 1 month after CO intoxication, the regions with significantly altered DTI and DKI indices had extended to the splenium of the corpus callosum, bilateral anterior and posterior limbs of the IC, bilateral anterior corona radiata, superior corona radiata, posterior corona radiata, and bilateral inferior longitudinal fasciculus. At 3 months after CO intoxication, the regions with significantly altered DTI and DKI indices had extended farther toward the subcortical areas and involved the bilateral forceps major; however, the RD, RK, and MK in the bilateral IC were partially normalized. At 9 months after CO intoxication, the regions with significantly altered DTI and DKI indices further involved the body of the corpus callosum and had extended farther into the subcortical areas; however, the FA, RK, and MK of the bilateral IC had normalized further, as shown in Figs 2 and 3 and Online Figs $1-5$. In patients without WMLs, the voxelwise comparison did not reveal significant change of DTI and DKI indices within 9 months after CO intoxication.

\section{Receiver Operating Characteristic Analysis}

The receiver operating characteristic analysis for all patients at 1 week after $\mathrm{CO}$ intoxication showed that areas under the curve of $>0.7$ were observed for $\mathrm{FA}$ in the left $\mathrm{CP}$; $\mathrm{AD}$ and $\mathrm{AK}$ in the bilateral IC; $\mathrm{AD}$ and $\mathrm{AK}$ in the right frontal WM (FWM); and RD, $\mathrm{MD}$, and $\mathrm{MK}$ in the left FWM. Notably, the area under the curve for MK in the left FWM was $>0.8$, and the areas under the curve for AK in the right IC and right FWM were $>0.9$ (Table 2). In addition, the longitudinal comparisons of DTI and DKI indices in these regions between patients with and without DNS are shown in On-line Figs 6-12. 


\section{DISCUSSION}

To the best of our knowledge, this is the first study in which voxelwise DKI analysis was applied to a longitudinal investigation of WM injuries in patients from 1 week to 9 months after CO intoxication. Unlike in previous studies, we divided the patients into 2 subgroups according to WML development after $\mathrm{CO}$ intoxication. The results demonstrated different progressions of WM injuries in the 2 subgroups, and all patients without WMLs did not have DNS, whereas 5 of the 11 (45\%) patients with WMLs developed DNS after CO intoxication. This finding suggests that DNS may be associated with WMLs. In addition, a previous study reported that dysfunctions of the GP and dopamine transporter may be associated with poor cognitive function and the presence of Parkinsonian features. ${ }^{8}$ In another study, a patient with GP involvement did not have Parkinsonism, though a patient with only WMLs $\operatorname{did} .{ }^{23}$ In the present study, only 3 of the 12 patients (25\%) with GP lesions were found to have
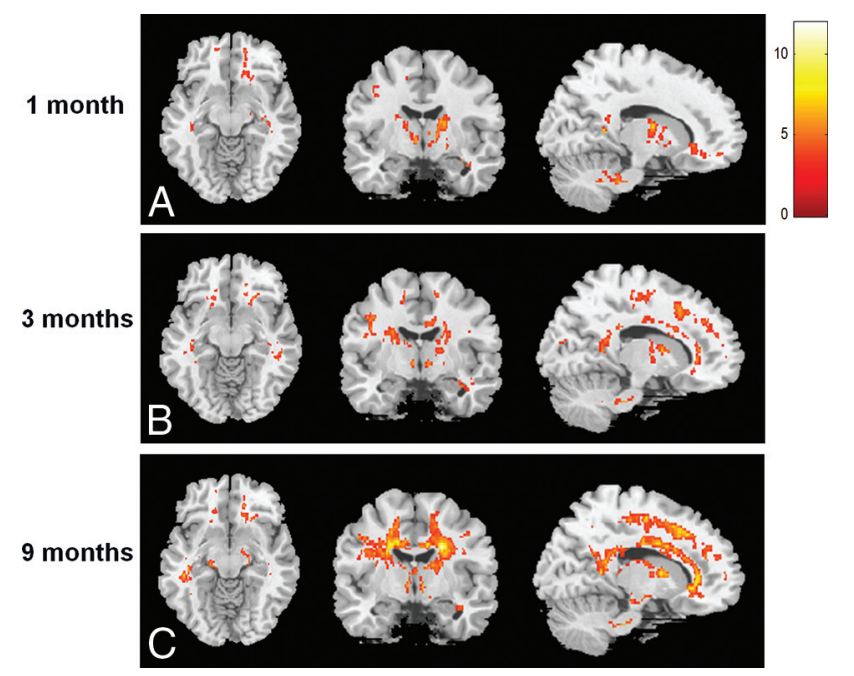

FIG 3. The voxelwise comparison of MK values between patients with WMLs and healthy controls. The yellow-to-red areas indicate regions with values that were significantly decreased in the patients, and the color bar on the right-hand side indicates $T$ values.

Table 2: The ROC analysis of diffusional indices for CP, IC, and FWM in differentiating patients with DNS from those without DNS at 1 week after CO intoxication ${ }^{\mathrm{a}}$

\begin{tabular}{|c|c|c|c|c|}
\hline $\begin{array}{l}\text { Brain Regions (MNI } \\
\text { Coordinates) (mm) }\end{array}$ & DNS & Non-DNS & Controls & $\begin{array}{c}\text { AUC } \\
\text { (DNS vs } \\
\text { non-DNS) }\end{array}$ \\
\hline \multicolumn{5}{|l|}{ Lt. CP $(-14,-14,-12)$} \\
\hline FA (mean) & $0.52 \pm 0.03$ & $0.56 \pm 0.06$ & $0.60 \pm 0.05$ & .7394 \\
\hline \multicolumn{5}{|l|}{ Rt. IC $(17,-10,0)$} \\
\hline $\mathrm{AD}$ (mean) & $1.38 \pm 0.08$ & $1.49 \pm 0.14$ & $1.49 \pm 0.08$ & .7394 \\
\hline AK (mean) & $0.87 \pm 0.05$ & $0.74 \pm 0.08$ & $0.80 \pm 0.03$ & $.9156^{b}$ \\
\hline \multicolumn{5}{|l|}{ Lt. IC $(-17,-10,0)$} \\
\hline $\mathrm{AD}$ (mean) & $1.38 \pm 0.06$ & $1.49 \pm 0.13$ & $1.48 \pm 0.11$ & .7762 \\
\hline AK (mean) & $0.89 \pm 0.06$ & $0.80 \pm 0.08$ & $0.81 \pm 0.08$ & .7113 \\
\hline \multicolumn{5}{|l|}{ Rt. FWM $(14,31,-12)$} \\
\hline $\mathrm{AD}$ (mean) & $1.30 \pm 0.03$ & $1.18 \pm 0.14$ & $1.19 \pm 0.14$ & .7436 \\
\hline AK (mean) & $1.03 \pm 0.04$ & $0.86 \pm 0.12$ & $0.83 \pm 0.09$ & $.9057^{b}$ \\
\hline \multicolumn{5}{|l|}{ Lt. FWM (-14, 31, -12) } \\
\hline $\mathrm{RD}$ (mean) & $0.78 \pm 0.04$ & $0.74 \pm 0.07$ & $0.62 \pm 0.07$ & .7436 \\
\hline $\mathrm{MD}$ (mean) & $0.94 \pm 0.03$ & $0.94 \pm 0.05$ & $0.90 \pm 0.06$ & .7299 \\
\hline MK (mean) & $0.98 \pm 0.06$ & $0.86 \pm 0.07$ & $0.89 \pm 0.12$ & $.8627^{\mathrm{b}}$ \\
\hline
\end{tabular}

Note:-AUC indicates area under the curve; ROC, receiver operating characteristic; Lt., left; Rt., right; MNI, Montreal Neurological Institute.

a The unit for $A D, R D$, and $M D$ is $10^{-3} \mathrm{~mm}^{2} / \mathrm{s}$.

${ }^{\mathrm{b}} \mathrm{P}<.05$.
DNS, indicating a stronger relationship between DNS and WMLs alone than between DNS and a GP lesion alone.

In patients with WMLs, significant WM injuries were initially detected (at 1 week after CO intoxication) in the pons, bilateral CP (anterior to the substantia nigra), bilateral IC, genu of the corpus callosum, and bilateral anterior prefrontal WMs. Consistent with a previous study, ${ }^{19}$ the WM injuries broadly reflected the alterations of dopaminergic pathways that may be responsible for Parkinsonian symptoms in patients with WMLs. In addition, the WM injuries gradually progressed to the subcortical and periventricular WM areas at 1 month and consistently deteriorated from 3 to 9 months. These results further suggest that the WM injuries in the subcortical brain regions may be associated with neuropsychiatric symptoms observed in patients with WMLs at the chronic stage. Similar to a previous study, ${ }^{9}$ the present study found that the changes in the DKI indices of the bilateral IC had normalized somewhat between 3 and 9 months after CO intoxication. The slight normalization of DKI indices suggests that the WM integrity of the bilateral IC was partially recovered between 3 and 9 months after CO intoxication.

In general, early WM injuries detected in this study were consistent with those reported in previous studies, ${ }^{18,19}$ though the discrepancy may likely result from the grouping strategy used in this study, in which patients were separated into WML and non-WML subgroups. This study further revealed that diffusional kurtosis-related indices outperformed diffusion tensor-related indices in differentiating patients with DNS from those without them and that the patients with DNS had significantly higher diffusional kurtosis values than those without them at 1 week after CO exposure. These findings suggest that early WM changes in the dopaminergic pathways were different between patients with and without DNS and that patients with DNS had a greater restriction of water diffusion in the WM tissues than the patients without DNS, likely due to more cytotoxic edema (hypoxia) at the early stage of CO intoxication.

Moreover, the results of this study may reflect the pathophysiologic mechanism of CO-induced WM injuries. First, inhalation of $\mathrm{CO}$ is known to cause hypoxia or oxidative stress in tissues that may lead to cytotoxic edema as a result of influx of calcium ions into the cells. ${ }^{1}$ As mentioned above, this study significantly demonstrated higher kurtosis values of WM tissues in patients with DNS than in those without DNS at 1 week, suggesting that patients with DNS may have undergone worse hypoxia than those without them. Second, free CO in plasma would gradually lead to subsequent inflammation in the tissues. ${ }^{24,25}$ This study shows that patients with WMLs exhibit significantly increased $\mathrm{RD}$ and unchanged $\mathrm{AD}$ in many WM regions between 1 week and 3 months, suggestive of WM demyelination due to the production of reactive oxygen species and lipid peroxidation at the subacute stage. $^{26}$ Third, ongoing inflammation and hypoxia would eventually lead to apoptosis and necrosis of tissues. ${ }^{24,25}$ This study demonstrates a significant increase 
in diffusivity and significant decreases in diffusion anisotropy and kurtosis in widespread WM tissues at 9 months after CO intoxication. These findings suggest that the ongoing inflammation eventually leads to axonal loss and increased microstructural complexity in WM tissues at the chronic stage. Nevertheless, in patients without WMLs, no significant change of diffusion indices was noted during the 9 months following CO intoxication, though some of these patients had GP lesions. We speculate that the WM may remain intact with relatively slight CO intoxication and that DNS was more closely related to WM changes than to GP lesions in patients with $\mathrm{CO}$ intoxication.

We acknowledge that this study has some limitations. First, the small sample size may have introduced statistical errors in the results. Second, the DKI data were acquired with only 2 high bvalues; therefore, the results may have been affected by the choice of b-values. ${ }^{27}$ Third, some patients did not undergo all DKI acquisitions because of severe coma, loss of contact, or drop-out, and the incomplete datasets may have affected the statistical results. Finally, the control group underwent only $1 \mathrm{MR}$ imaging without follow-up; therefore, the WM injuries found within 9 months of $\mathrm{CO}$ intoxication may have been affected by aging.

\section{CONCLUSIONS}

Voxelwise DKI analysis revealed longitudinal WM changes in patients after CO intoxication. DNS developed in $45 \%$ of patients with WMLs but in none of the patients without WMLs. The voxelwise comparisons also showed that patients without WMLs did not exhibit progressive WM changes after CO intoxication, whereas patients with WMLs had early WM injuries to the dopaminergic pathways at 1 week after $\mathrm{CO}$ intoxication, with WM injuries progressing toward the subcortical and periventricular regions from 1 to 9 months. The values of AK and MK in the IC and FWM performed better than other diffusion indices in differentiating patients with DNS from those without them. We conclude that voxelwise DKI analysis was helpful for assessing longitudinal WM injuries and in predicting the prognosis of patients after CO intoxication.

Disclosures: Ping-Hong Lai-RELATED: Grant: Ministry of Science and Technology, Taiwan (106-2314-B-075B-002-MY3)

\section{REFERENCES}

1. Weaver LK. Clinical practice: carbon monoxide poisoning. $N$ Engl J Med 2009;360:1217-25 CrossRef Medline

2. Ng PC, Long B, Koyfman A. Clinical chameleons: an emergency medicine focused review of carbon monoxide poisoning. Intern Emerg Med 2018;13:223-29 CrossRef Medline

3. Weaver LK, Hopkins RO, Chan KJ, et al. Hyperbaric oxygen for acute carbon monoxide poisoning. $N$ Engl J Med 2002;347:1057-67 CrossRef Medline

4. Weaver LK. Hyperbaric oxygen therapy for carbon monoxide poisoning. Undersea Hyperbar M 2014;41:339-54 Medline

5. Weaver LK, Valentine KJ, Hopkins RO. Carbon monoxide poisoning: risk factors for cognitive sequelae and the role of hyperbaric oxygen. Am J Resp Crit Care 2007;176:491-97 CrossRef Medline

6. Hou XM, Ma LL, Wu LH, et al. Diffusion tensor imaging for predicting the clinical outcome of delayed encephalopathy of acute carbon monoxide poisoning. Eur Neurol 2013;69:275-80 CrossRef Medline

7. Lo CP, Chen SY, Chou MC, et al. Diffusion-tensor MR imaging for evaluation of the efficacy of hyperbaric oxygen therapy in patients with delayed neuropsychiatric syndrome caused by carbon monoxide inhalation. Eur J Neurol 2007;14:777-82 CrossRef Medline
8. Chang CC, Chang WN, Lui CC, et al. Clinical significance of the pallidoreticular pathway in patients with carbon monoxide intoxication. Brain 2011;134(Pt 12):3632-46 CrossRef Medline

9. Chang CC, Chang WN, Lui CC, et al. Longitudinal study of carbon monoxide intoxication by diffusion tensor imaging with neurospsychiatric correlation. J Psychiatr Neurosci 2010;35:115-25 CrossRef Medline

10. Lin $\mathrm{WC}, \mathrm{Lu} \mathrm{CH}$, Lee $\mathrm{YC}$, et al. White matter damage in carbon monoxide intoxication assessed in vivo using diffusion tensor MR imaging. AJNR Am J Neuroradiol 2009;30:1248-55 CrossRef Medline

11. Chen PC, Chen MH, Chen HL, et al. Callosal damage and cognitive deficits in chronic carbon monoxide intoxication: a diffusion tensor imaging study. J Neurol Sci 2015;355:101-07 CrossRef Medline

12. Beppu T, Fujiwara S, Nishimoto H, et al. Fractional anisotropy in the centrum semiovale as a quantitative indicator of cerebral white matter damage in the subacute phase in patients with carbon monoxide poisoning: correlation with the concentration of myelin basic protein in cerebrospinal fluid. J Neurol 2012;259:1698-705 CrossRef Medline

13. Fujiwara $S$, Beppu $T$, Nishimoto $H$, et al. Detecting damaged regions of cerebral white matter in the subacute phase after carbon monoxide poisoning using voxel-based analysis with diffusion tensor imaging. Neuroradiology 2012;54:681-89 CrossRef Medline

14. Basser PJ, Mattiello J, Lebihan D. MR diffusion tensor spectroscopy and imaging. Biophys J 1994;66:259-67 CrossRef Medline

15. Jensen JH, Helpern JA, Ramani A, et al. Diffusional kurtosis imaging: the quantification of non-Gaussian water diffusion by means of magnetic resonance imaging. Magn Reson Med 2005;53: 1432-40 CrossRef Medline

16. Veraart J, Poot DH, Van Hecke W, et al. More accurate estimation of diffusion tensor parameters using diffusion kurtosis imaging. Magn Reson Med 2011;65:138-45 CrossRef Medline

17. Arab A, Wojna-Pelczar A, Khairnar A, et al. Principles of diffusion kurtosis imaging and its role in early diagnosis of neurodegenerative disorders. Brain Res Bull 2018;139:91-98 CrossRef Medline

18. Tsai PH, Chou MC, Chiang SW, et al. Early white matter injuries in patients with acute carbon monoxide intoxication: a tract-specific diffusion kurtosis imaging study and STROBE compliant article. Medicine (Baltimore) 2017;96:e5982 CrossRef Medline

19. Chou MC, Lai PH, Li JY. Early white matter injuries associated with dopamine transporter dysfunction in patients with acute CO intoxication: a diffusion kurtosis imaging and Tc-99m TRODAT-1 SPECT study. Eur Radiol 2018 Aug 24. [Epub ahead of print] CrossRef Medline

20. Choi IS. Delayed neurologic sequelae in carbon-monoxide intoxication. Arch Neurol 1983;40:433-35 CrossRef Medline

21. Fan W, Zheng W, Chen X. A Better Characterization of brain damage in carbon monoxide intoxication assessed in vivo using diffusion kurtosis imaging. In: Proceedings of the Annual Meeting of the International Conference on Biological Sciences and Technology, Guangzhou, China. January 8-10, 2016:263-68

22. Mori S, Oishi K, Jiang HY, et al. Stereotaxic white matter atlas based on diffusion tensor imaging in an ICBM template. Neuroimage 2008;40:570-82 CrossRef Medline

23. Sohn YH, Jeong Y, Kim HS, et al. The brain lesion responsible for parkinsonism after carbon monoxide poisoning. Arch Neurol 2000; 57:1214-18 CrossRef Medline

24. Piantadosi CA, Zhang J, Levin ED, et al. Apoptosis and delayed neuronal damage after carbon monoxide poisoning in the rat. Exp Neurol 1997;147:103-14 CrossRef Medline

25. Cronje FJ, Carraway MS, Freiberger JJ, et al. Carbon monoxide actuates $\mathrm{O}$-2-limited heme degradation in the rat brain. Free Radic Biol Med 2004;37:1802-12 CrossRef Medline

26. Thom SR, Bhopale VM, Han ST, et al. Intravascular neutrophil activation due to carbon monoxide poisoning. Am J Resp Crit Care 2006;174:1239-48 CrossRef Medline

27. Chou MC, Ko CW, Chiu YH, et al. Effects of b value on quantification of rapid diffusion kurtosis imaging in normal and acute ischemic brain tissues. J Comput Assist Tomogr 2017;41:868-76 CrossRef Medline 